\title{
THE RADIATION FIELD IS A FOURIER INTEGRAL OPERATOR
}

\author{
ANTÔNIO SÁ BARRETO AND JARED WUNSCH
}

\section{INTRODUCTION}

In this note, we exhibit explicitly the form of the "radiation field" of F. G. Friedlander on two different types of manifolds: scattering manifolds, and asymptotically hyperbolic manifolds. The former class consists of manifolds with ends that look asymptotically like the large ends of cones, and includes a large class of asymptotically Euclidean spaces, while the latter consists of spaces that resemble the hyperbolic space at infinity, and includes quotients of hyperbolic space by certain groups of motion. In both cases we assume that there are no trapped geodesics. The radiation field is a measurement of the (rescaled) asymptotic behavior of solutions to the wave equation, viewed from the point of view of a rescaled time coordinate which in the asymptotically Euclidean setting is simply $s=t-r$, and restricted to the sphere at infinity. In particular, in $\mathbb{R}^{n}$, we define

$$
\mathcal{R}_{+}\left(s, \theta, z^{\prime}\right)=\lim _{r \rightarrow \infty} r^{(n-1) / 2} V\left(s+r, r \theta, z^{\prime}\right)
$$

where $V\left(t, z, z^{\prime}\right)=(\cos t \sqrt{\Delta}, \sin t \sqrt{\Delta} / \sqrt{\Delta})$ is the solution operator to the wave equation. Friedlander showed in [5] that $\mathcal{R}_{+}$is in fact a a translation representation of the wave group in the sense introduced by Lax and Phillips [12]. (Sá Barreto [21, 20] subsequently showed the unitarity of this map.)

In this paper, we show that the radiation field $\mathcal{R}_{+}$on a manifold $X$ which is either a scattering manifold or an asymptotically hyperbolic manifold, has as its Schwartz kernel a Lagrangian distribution, associated to the conic Lagrangian defined by the graph of a "sojourn relation" relating points in $T^{*} \stackrel{\circ}{X}$ to points in $T^{*}(\mathbb{R} \times \partial X)$, where $\partial X$ is the boundary at infinity. In the simple example where $\stackrel{\circ}{X}$ is just Euclidean space, the graph of the sojourn relation maps $T^{*} \mathbb{R}^{n}$ to $T^{*}\left(\mathbb{R} \times S^{n-1}\right)$ roughly as follows: given $(z, \hat{\zeta}) \in S^{*}\left(\mathbb{R}^{n}\right)$, let $\gamma$ be the unique unit speed geodesic passing through it. We map $(z, \zeta)$ to the base point $(s, \theta) \in \mathbb{R} \times S^{n-1}$ where $s$ is given by the "sojourn time" or the limit of $t-r$ along the geodesic, and $\theta$ is the asymptotic direction in $S^{n-1}$. The fiber variables then measure the angle of contact the geodesic makes with $S^{n-1}$ in a way made precise below. This sojourn relation on scattering manifolds was previously investigated by Hassell-Wunsch [8]

The first author acknowledges support from NSF grant DMS-0140657 and the second author from grant DMS-0323021. 
in the context of the fundamental solution to the Schrödinger equation on scattering manifolds; it is also closely related to the sojourn time defined by Guillemin in [7] in the study of the high frequency asymptotics of the scattering matrix. Note that the sojourn time $s$ is more or less just the "Busemann function" used in differential geometry.

In the special case in which we locally have a finitely many geodesics $\gamma_{n}(t)$ beginning at a point $z \in \stackrel{\circ}{X}$ with asymptotic direction $\theta$, the construction is simpler: $\mathcal{R}_{+}$is a conormal distribution with respect to the hypersurfaces $s=S_{n}(z, \theta) \equiv \lim t-r\left(\gamma_{n}(t)\right)$. In this case, Fourier transforming yields a simple result about the high-frequency asymptotics of the scattering Poisson operator (better known, in the asymptotically hyperbolic case, as the Eisenstein function). This result is a weak generalization of a result of Guillemin [7, who proved on compactly supported perturbations of Euclidean space that not only do the scattering operator and Poisson operator have the form discussed here, but a composition of FIO's gives the asymptotics of the scattering matrix as well. We are unable to perform this composition owing to the local nature of our results in $z$, the variable in $\mathbb{R}^{n}$ (the location of the initial pole of the fundamental solution). Our results are also weaker in the sense that we obtain only distributional asymptotics of the Poisson operator, i.e. we must mollify by convolution with the inverse Fourier transform of a compactly supported cutoff in order to describe the asymptotics. On the other hand the results presented here are novel insofar as we do permit global perturbations of the metric and folded sojourn relations, and we treat the asymptotically hyperbolic case as well.

In the case of obstacle, rather than metric, scattering, results relating the scattering matrix and the sojourn time were initially obtained by Majda [13, and used in the solution of inverse problems. For further applications of sojourn-time methods in inverse obstacle scattering, see the survey by Petkov and Stoyanov [18. Similar results to ours in the case of semiclassical scattering on $\mathbb{R}^{n}$ have also been obtained by Robert and Tamura [19]; more recently, Alexandrova [1] has extended these results to show that even if the Hamilton flow is degenerate, the scattering matrix is a semiclassical FIO.

We now discuss simple examples in which the Poisson operator is explicitly known and the appearance of the sojourn time in the high-frequency behavior is clear.

Example 1. On $\mathbb{R}^{n}$, the kernel of the scattering-theoretic Poisson operator, evaluated at $(z, \theta) \in \mathbb{R} \times S^{n-1}$, is just

$$
\left(\frac{i \lambda}{2 \pi}\right)^{(n-1) / 2} e^{i \lambda \theta \cdot z}
$$

(see [16]); this is the operator mapping high-frequency incoming scattering data to a generalized eigenfunction with eigenvalue $\lambda^{2}$ (see $\$ 2.2$ for a precise definition.). 
On the other hand, there is a unique geodesic beginning at $z$ with asymptotic direction $\theta$ : it is just $z+t \theta$. The sojourn time along the geodesic is

$$
\lim |t-z+t \theta|=-\theta \cdot z,
$$

hence exactly the phase of the adjoint of the Poisson operator.

Example 2. The kernel of the Poisson operator on hyperbolic space is better known as the "Eisenstein function;" it is given by

$$
E\left(\frac{n}{2}+i \lambda, y, z\right)=\lim _{x \rightarrow 0^{+}} x^{-n / 2-i \lambda} R\left(\frac{n}{2}+i \lambda, x, y, z\right)
$$

where $R(\lambda)$ is the resolvent, normalized to be $\left(\Delta-\lambda^{2}-\frac{n^{2}}{4}\right)^{-1}$ and where we work in the usual coordinates on the half-space with defining function $x$. On $\mathbb{H}^{3}$, the resolvent is just (see equation (6.8) of [14]):

$$
R\left(\frac{n}{2}+i \lambda\right)=C \frac{e^{-i \lambda \delta}}{\sinh \delta}
$$

where $\delta$ is the hyperbolic distance. The phase is thus $-\delta$, which is asymptotic to

$$
-\log \frac{x^{2}+\left(x^{\prime}\right)^{2}+\left(y-y^{\prime}\right)^{2}}{x x^{\prime}}
$$

hence, switching primed and unprimed variables, the phase of $E\left(\frac{n}{2}+i \lambda, y^{\prime}, z\right)$ is just

$$
\phi\left(x, y, y^{\prime}\right) \equiv-\log \frac{x^{2}+\left(y-y^{\prime}\right)^{2}}{x} .
$$

Now we compare this phase to the sojourn time. Given a point $z \in \mathbb{H}^{3}$ and $y^{\prime} \in \partial \mathbb{H}^{3}$, there is a unique geodesic $\gamma(t)$ starting at $z$, and approaching $y^{\prime}$ as $t \rightarrow \infty$ (this is the analogue of the "asymptotic direction" $\theta \in S^{n-1}$ in the Euclidean case). We now define the sojourn time for this geodesic as

$$
S=S\left(z, y^{\prime}\right)=\lim t+\log x(\gamma(t))
$$

Using translation invariance in the boundary variables, it suffices to compute with $y^{\prime}=0$. A unit speed geodesic from an arbitrary point $(x, y) \in \mathbb{H}^{3}$ to $(0,0) \in \partial \mathbb{H}^{3}$ can be parametrized as

$$
x=C \operatorname{sech}\left(t+t_{0}\right), y=C \tanh \left(t+t_{0}\right)+D,
$$

whence we compute $\phi(x, y, 0)=-\log 2-\log C+t_{0}$. On the other hand, the sojourn time along such a geodesic is

$$
S(x, y, 0) \equiv \lim _{t \rightarrow+\infty} t+\log x(t)=\log 2+\log C-t_{0},
$$

hence agrees with minus the phase $\phi(x, y, 0)$. 


\section{SCATTERING MANIFOLDS}

2.1. Radiation field. Let $X$ be a $\mathcal{C}^{\infty}$ compact manifold with a boundary. A scattering metric (defined originally by Melrose [17) is a metric on a manifold with boundary that can be brought to the form

$$
g=\frac{d x^{2}}{x^{4}}+\frac{h}{x^{2}}
$$

with $x$ a boundary defining function and $h$ a smooth tensor that restricts to $x=0$ to give a metric $h_{0}$ on $\partial X$. This form is modeled after the metric on asymptotically Euclidean space, radially compactified. It was shown by Joshi-Sá Barreto 9] that there is a normal form for a scattering metric: in which a neighborhood of the boundary admits a product decomposition $[0, \epsilon) \times \partial X$ with local coordinates $(x, y)$ in which $g$ takes the form (2.1) with $h=h(x, y, d y)$ a smooth family in $x$ of metrics on $\partial X$. Note that in these product coordinates, the rays $y=$ constant are (infinitely extended) geodesics. We henceforth assume that our metric is in normal form. We further make the geometric assumption that there are no trapped rays in $\stackrel{\circ}{X}$.

Following Friedlander [5] we define the forward radiation field on a scattering manifolds by

$$
\mathcal{R}_{+}\left(f_{1}, f_{2}\right)(s, y)=\left(x^{-(n-1) / 2} D_{t} H u\right)\left(s+x^{-1}, x, y\right) \Gamma_{x=0}
$$

where $H=H(t)$ is the Heaviside function and $u=u(t, x, y)$ is the solution to

$$
\square u=0, \quad\left(u, D_{t} u\right) \Upsilon_{t=0}=\left(f_{1}, f_{2}\right) .
$$

Friedlander showed that $\mathcal{R}_{+}\left(f_{1}, f_{2}\right) \in \mathcal{C}^{\infty}(\mathbb{R} \times \partial X)$ provided $f_{1}, f_{2}$ are smooth and compactly supported in $\stackrel{\circ}{X}$. Thus the Schwartz kernel

$$
\mathcal{R}_{+}(s, y, z)
$$

is defined on $\mathbb{R} \times \partial X$; here we have used coordinates $(s, y, z)$ on $\mathbb{R} \times \partial X \times \stackrel{\circ}{X}$. First we will show that the radiation field is an FIO and then, under some nondegeneracy conditions, we will compute its symbol. We use coordinates on $T^{*}(\mathbb{R} \times \partial X \times \stackrel{\circ}{X})$ defined by the canonical one-form

$$
s d \sigma+y d \eta+z d \zeta,
$$

and will employ the notation $\hat{\zeta}=\zeta /|\zeta|$.

Theorem 2.1. Let $X$ be a nontrapping scattering manifold. Then

$$
\mathcal{R}_{+} \in\left(I^{1}\left(\mathbb{R} \times \partial X \times \stackrel{\circ}{X}, \Lambda^{\prime} ; \Omega^{1 / 2}\right), I^{0}\left(\mathbb{R} \times \partial X \times \stackrel{\circ}{X}, \Lambda^{\prime} ; \Omega^{1 / 2}\right)\right),
$$


where $\Omega$ is the density bundle on $\mathbb{R} \times \partial X \times \stackrel{\circ}{X}$, and $\Lambda_{ \pm}^{\prime}$ is the conic Lagrangian manifold associated to the graph of the "sojourn relation":

$$
\begin{gathered}
\Lambda_{ \pm}=\Lambda_{+} \cup \Lambda_{-}, \\
\Lambda_{ \pm}=\left\{z, \zeta, s=\lim _{t \rightarrow \pm \infty} t-x^{-1}\left(\exp _{z}(t \hat{\zeta})\right), \sigma= \pm|\zeta|\right. \\
\left.y=\lim _{t \rightarrow \pm \infty} y\left(\exp _{z}(t \hat{\zeta})\right), \eta_{i}=\lim _{t \rightarrow \pm \infty} \pm|\zeta|\left(h_{0}\right)_{i j}\left(d y^{j} / d x\right)\left(\exp _{z}(t \hat{\zeta})\right)\right\} .
\end{gathered}
$$

Proof. We know from the non-trapping assumption and from Theorem 1.1 of [3] that the kernel of $\cos t \sqrt{\Delta}$, which is the solution to (2.2) with $f_{1}=\delta(z)$ and $f_{2}=0$, a sum of forward and backward parts (corresponding to a choice of \pm in our notation below), each of which is a Lagrangian distribution in $I^{-\frac{1}{4}}\left(\mathbb{R} \times X \times X ; \mathcal{C}_{ \pm}\right)$, where

$$
\begin{gathered}
\mathcal{C}_{ \pm}=\left\{\left(t, z, z^{\prime}, \tau, \zeta, \zeta^{\prime}\right):(z, \zeta),\left(z^{\prime}, \zeta^{\prime}\right) \in T^{*} X \backslash 0,(t, \tau) \in T^{*} \mathbb{R} \backslash 0,\right. \\
\left.\tau= \pm \sqrt{L(z, \zeta)},(z, \zeta)=\Phi^{ \pm t}\left(z^{\prime}, \zeta^{\prime}\right)\right\}
\end{gathered}
$$

Here $L(z, \zeta)$ denotes the symbol of $\Delta$ and $\Phi^{t}\left(z^{\prime}, \zeta^{\prime}\right)$ denotes the flow along $H_{L}$ in $T^{*} X \backslash 0$. We want to understand what happens to the kernel of $\cos t \sqrt{\Delta}$ if one first makes the change $s=t-1 / x$ and then takes the limit $x \rightarrow 0$. So we need to understand the effect of these operations on $\mathcal{C}_{ \pm}$and the distribution associated with it.

As in 21, let $P=x^{-2-(n-1) / 2} \square x^{(n-1) / 2}$ and change variables, replacing $t$ by $s=t-1 / x$. We find as in 21] that

$P=2 \frac{\partial}{\partial x} \frac{\partial}{\partial s}+x^{2} \frac{\partial^{2}}{\partial x^{2}}-\Delta_{h}+A \frac{\partial}{\partial s}+\left(2 x+x^{2} A\right) \frac{\partial}{\partial x}+\left(\frac{n-1}{2}\right)\left(\frac{3-n}{2}+x A\right)$

with $A(x, y)=\partial_{x} \log |h|^{1 / 2}$ and $\Delta_{h}$ is the nonnegative Laplacian on $\partial X$ with respect to the metric $h$. The symbol of $P$ in these coordinates is given by

$$
p=-2 \xi \sigma-x^{2} \xi^{2}-h(x, y, \eta)
$$

and the Hamilton vector field by

$$
H_{p}=-2\left(\sigma+x^{2} \xi\right) \frac{\partial}{\partial x}-2 \xi \frac{\partial}{\partial s}+\left(2 x \xi^{2}+\frac{\partial h}{\partial x}\right) \frac{\partial}{\partial \xi}-H_{h}
$$

$((z, \zeta)$, the coordinates in the right factor, are left invariant by the flow).

If $V_{0}=x^{-\frac{n-1}{2}} U_{0}$, the equation (2.2) with initial data $f_{1}=\delta(z)$ and $f_{2}=0$ becomes

$$
P V_{0}=0, \quad V_{0} \Upsilon_{s=-\frac{1}{x}}=x^{-\frac{n-1}{2}} \delta(z), \quad D_{t} V_{0} \uparrow_{s=-\frac{1}{x}}=0, \quad x>0
$$

We remark that we are ignoring half-density factors, as they are irrelevant to this construction. Notice that the operator $P$ extends to $x \leq 0$ as a strictly hyperbolic differential operator $\widetilde{P}$ and that $s=-\frac{1}{x}$ is a space-like surface for $\widetilde{P}$. One can think of this as being an extension of $P$ to the double manifold 
$X^{2}=(X \sqcup X) / \partial X$. Therefore it follows from the existence of a fundamental solution to the Cauchy problem for strictly hyperbolic operators, see for example Theorem 5.1.6 of [2], that the solution to (2.4), with $P$ replaced by $\widetilde{P}$, is a Lagrangian distribution $\widetilde{V_{0}}$ of class

$$
\widetilde{V_{0}} \in I^{-\frac{1}{4}}(\mathbb{R} \times X \times \stackrel{\circ}{X} ; \mathcal{L}),
$$

where $\mathcal{L}$ denotes the Lagrangian in $T^{*}(\mathbb{R} \times X \times \stackrel{\circ}{X})$ obtained by flowing $N^{*}\left\{z=z^{\prime}, \quad t=0\right\} \cap \Sigma_{p} \subset T^{*}(\mathbb{R} \times X \times \stackrel{\circ}{X})$ along the integral curves of $H_{\widetilde{p}}$, where $\widetilde{p}$ is the principal symbol of $\widetilde{P}$, and $\Sigma_{\widetilde{p}}$ denotes the characteristic variety of $\widetilde{P}$. By the uniqueness of solutions to the Cauchy problem, the restriction of $\widetilde{V_{0}}$ solution to $x>0$ is equal to $V_{0}$, the solution to (2.4).

We remark that the extension $\widetilde{P}$, and consequently the definition of $\mathcal{L}$ in $\{x<0\}$, are not unique. However the extensions to $\{x=0\}$ are.

Since $N^{*}(\{x=0\}) \cap \mathcal{L}=0, U \equiv \widetilde{V_{0}} \uparrow_{x=0}$ is a Lagrangian distribution of order 1 on $\mathbb{R} \times \partial X \times \stackrel{\circ}{X}$ with respect to $\mathcal{L} \uparrow_{x=0}$. Hence for any compactly supported distribution $u, \mathcal{R}_{+}(u, 0)=D_{t} \widetilde{U} u$ where $\widetilde{U}$ is a Lagrangian of order 0 with respect to $\mathcal{L} \uparrow_{x=0}$.

To identify $\mathcal{L} \uparrow_{x=0}$ geometrically we observe that under the flowout of $H_{p}$, $\sigma$ is conserved, hence by homogeneity we need only consider $\sigma= \pm 1$ (note that $\sigma \neq 0$ on $\mathcal{L} \backslash 0$ ). Let $\mathcal{L}_{ \pm}$denote the two components corresponding to different signs of $\sigma$. By definition, we certainly have $s=\lim _{t \rightarrow \pm \infty}(t-$ $x^{-1}$ ), and $y$ is the limiting location of geodesic flow (forward or backward according to $\operatorname{sgn} \sigma)$ in $\partial X$. Furthermore, setting $\sigma= \pm 1$ we have $d y^{i} / d x=$ $\left(-2 h^{i j} \eta_{j}\right) /\left(-2\left( \pm 1+x^{2} \xi\right)\right)$, which approaches $\pm h^{i j} \eta_{j}$ as $x \rightarrow 0$. Thus the restriction of $\mathcal{L}_{ \pm}$equals $\Lambda_{ \pm}$as defined in the statement of the theorem.

Similarly, solving

$$
P U_{1}=0, \quad U_{1} \Upsilon_{t=0}=0, \quad D_{t} U_{0} \Upsilon_{t=0}=\delta(z)
$$

gives a Lagrangian of order -1 when restricted to the boundary. Hence for any distributions $f_{0}, f_{1}$,

$$
\mathcal{R}_{+}\left(f_{0}, f_{2}\right)=\left(D_{t} U_{0} f_{0}, D_{t} U_{1} f_{1}\right) \Gamma_{x=0}
$$

is a FIO of the asserted kind.

It remains to calculate the symbol of $\mathcal{R}_{+}$and for this we need some extra assumptions. So we suppose further that for all $z$ contained in an open set $U_{1}$ in $\stackrel{\circ}{X}$, all $y$ contained in an open set $U_{2}$ in $\partial X$, there exist a finite number of unit speed geodesics $\gamma_{n}(z, y, t), n=1, \ldots, N$ such that

$$
\gamma_{n}(z, y, 0)=z, \quad \lim _{t \rightarrow+\infty} \gamma_{n}(z, y, t)=y,
$$

and such that the transformation

$$
\frac{\partial^{2} \gamma_{n}}{\partial y \partial t} \Gamma_{t=0}
$$


mapping

$$
T(\partial X) \rightarrow T(S(\stackrel{\circ}{X}))
$$

is invertible for all $n$, and $y \in U_{2}, z \in U_{1}$. Subject to these assumptions, the Lagrangian $\Lambda$ is projectable onto the $z, y$ variables. Letting

$$
S_{n}(z, y)=\lim _{t \rightarrow+\infty} t-x^{-1}\left(\gamma_{n}(z, y, t)\right)
$$

(the "sojourn times"), we then find that $\mathcal{R}_{+}\left(0, \delta_{z}\right)$ is conormal to the surfaces $s=S_{n}(z, y)$ in $\mathbb{R}_{s} \times U_{1} \times U_{2}$.

Theorem 2.2. Subject to the nondegeneracy assumptions above, the symbol of $\mathcal{R}_{+}$, evaluated at $(z, y, \sigma) \in N^{*}\left\{s=S_{n}\right\}$ equals

$$
\frac{1}{2} i^{k_{n}}\left|\frac{\partial y^{(n)}}{\partial \hat{\zeta}}\right|^{-1 / 2}\left|d s d h_{y} d g_{z}\right|^{1 / 2} \cdot\left(\sigma^{(n+1) / 2}, \sigma^{(n-1) / 2}\right) .
$$

where $k_{n}$ is the number of conjugate points encountered by $\gamma_{n}(z, y, t)$ with $t \in(0, \infty)$ and $\left|\partial y^{(n)}(z, y) / \partial \hat{\zeta}\right|$ is short for the Jacobian of the map

$$
S_{z}^{*}(\stackrel{\circ}{X}) \rightarrow \partial X
$$

given by the limit of geodesic flow, evaluated at the initial codirection of $\gamma_{n}$.

Note that we have written the Jacobian factor in the above form to emphasize the analogy with the differential scattering cross-section in Guillemin's results [7].

Proof. Let OG $\subset S^{*}(\stackrel{\circ}{X})$ denote the outgoing set, i.e. the set on which $d x / d t<0$ along the bicharacteristic flow. Owing to our nontrapping assumption, the cosphere bundle of a compact set $S^{*}(K) \subset S^{*}(\stackrel{\circ}{X})$ eventually maps into OG under the bicharacteristic flow after time $T \gg 0$. Let us fix such $K$ and $T$, with $K$ chosen such that our nondegeneracy assumption holds for all geodesics beginning in $K$ with limits some open set in $y$.

Net $Z$ be a compactly supported pseudodifferential operator with $\mathrm{WF}^{\prime} Z \subset$ OG, $W F^{\prime}(1-Z) \cap O G^{\prime}=\emptyset$, with $O^{\prime}$ a conic subset of OG with compact projection, chosen so that the flowout of $S^{*} K$ for time $T$ lies inside $O G^{\prime}$.

Let $Z_{j}^{\prime}$ be a microlocal partition of unity over $K$ such that for all $j$, and all $z \in \stackrel{\circ}{X}, \mathrm{WF}^{\prime} Z_{j}^{\prime} \cap \pi^{-1}(z)$ contains at most a single point such that the geodesic emanating from this point ends up at $y \in \partial X$. By our hypotheses on the nondegeneracy of geodesics, we may further choose $Z$ supported sufficiently close to $y$ that if $\left(z^{\prime}, \zeta^{\prime}\right)$ and $(z, \zeta)$ are canonical coordinates on the cotangent bundles of left and right factors, then $\left(\zeta^{\prime}, z\right)$ are coordinates on

$$
\Lambda_{ \pm} \cap \pi_{L}^{*} \mathrm{WF}^{\prime} Z \cap \pi_{R}^{*} \mathrm{WF}^{\prime} Z_{j}^{\prime} .
$$

We may further arrange, by working sufficiently close to $\partial X$, that there are no conjugate points for bicharacteristics beginning on $\mathrm{WF}^{\prime} Z$ : examination of the Hamilton flow in "scattering coordinates" shows that the tangent 
vectors to geodesics emanating from $\mathrm{WF}^{\prime} Z$ approach $-x^{2} \partial x$, and while the sectional curvature of a scattering manifold is $O\left(x^{2}\right)$, the sectional curvature of a plane containing $x^{2} \partial x$ is $O\left(x^{3}\right)$. Hence certainly the sectional curvature along a plane containing the tangent to the geodesic is $o\left(x^{2}\right)$. By a simple variant on the Rauch comparison theorem, this is sufficient to ensure the absence of conjugate points. (See [15] for a description of the bicharacteristic flow in scattering coordinates and, for instance Theorem 4.5.1 of [1] for an account of the relevant comparison theorem.)

Now let $W(t)$ denote the propagator for the wave group. The symbol of $W(T) Z_{j}^{\prime}$ is

$$
\frac{1}{2} \sigma\left(Z_{j}^{\prime}\right)(z, \zeta) i^{k_{n}}|d z d \zeta|^{1 / 2}\left(\begin{array}{cc}
1 & |\zeta|_{g}^{-1} \\
|\zeta|_{g} & 1
\end{array}\right)
$$

where $k_{n}$ is the number of conjugate points encountered. Changing to coordinates $\left(\zeta^{\prime}, z\right)$ gives

$$
\frac{1}{2} \pi_{R}^{*} \sigma\left(Z_{j}^{\prime}\right) i^{k_{n}}\left|\frac{\partial \zeta^{\prime}}{\partial \zeta}\right|^{-1 / 2}\left|d \zeta^{\prime} d z\right|^{1 / 2}\left(\begin{array}{cc}
1 & \left|\zeta^{\prime}\right|_{g}^{-1} \\
\left|\zeta^{\prime}\right|_{g} & 1
\end{array}\right) .
$$

By invariance under the bicharacteristic flow (see for instance Proposition 4.3.1 of [2]), the symbol of $\mathcal{R}_{+} Z$ is

$$
\frac{1}{2} \pi_{R}^{*} \sigma(Z)\left|\frac{\partial(s, y)}{\partial \zeta^{\prime}}\right|^{-1 / 2}\left|d s d y d z^{\prime}\right|^{1 / 2}(|\sigma|, 1) .
$$

We split the coordinate $\zeta^{\prime}$ into $|\zeta|^{\prime}, \hat{\zeta}^{\prime}$ (where the latter should really be regarded as $n-1$ components of $\hat{\zeta}^{\prime}$ ). By homogeneity of the flow we have $\partial s / \partial|\zeta|^{\prime}=1$, while $\partial y / \partial|\zeta|^{\prime}=0$, hence the symbol of $\mathcal{R}_{+} Z$ can in fact be written

$$
\frac{1}{2} \pi_{R}^{*} \sigma(Z)\left|\frac{\partial(y)}{\partial \hat{\zeta}^{\prime}}\right|^{-1 / 2}\left|d s d y d z^{\prime}\right|^{1 / 2}(|\sigma|, 1) .
$$

The difference

$$
\mathcal{R}_{+}(s) Z_{j}^{\prime}-\mathcal{R}_{+}(s-T) Z W(T) Z_{j}^{\prime}
$$

is a smoothing operator. Hence applying the calculus of FIO's to the above results and patching together the partition of unity $Z_{j}^{\prime}$, we find that the symbol of $\mathcal{R}_{+}$is given globally by (2.7).

2.2. Poisson operator. Let $P(\lambda)$ denote (the Schwartz kernel of) the Poisson operator, i.e. the operator such that for any $g \in \mathcal{C}^{\infty}(\partial X)$ there exists $u \in \mathcal{C}^{\infty}(X)$ with $\left(\Delta-\lambda^{2}\right) u=0$ and

$$
u=e^{i \lambda / x} x^{(n-1) / 2} g+e^{-i \lambda / x} x^{(n-1) / 2} g_{-}+u^{\prime}
$$

with $g^{\prime} \in \mathcal{C}^{\infty}(\partial X), u^{\prime} \in L^{2}(X ; g)$.

As a corollary of the results in the preceding section, we conclude the following: 
Proposition 2.3. Suppose as in the preceding proposition that there exist a finite number of nondegenerate geodesics $\gamma_{n}$ from $z$ to $y$. Let $\check{\phi} \in \mathcal{C}_{c}^{\infty}(\mathbb{R})$. Then as $|\lambda| \rightarrow \infty$,

$$
\phi(\lambda) * P(\lambda)^{*}(y, z) \sim \phi(\lambda) * \sum_{n=1}^{N} i^{k_{n}} e^{i \lambda S_{n}}\left(\frac{\lambda}{2 \pi i}\right)^{(n-1) / 2}\left|\frac{\partial y^{(n)}}{\partial \hat{\zeta}}\right|^{-1 / 2}
$$

Proof. We simply use the fact that

$$
P(\lambda)^{*}(y, z)=-2 \mathcal{F} \mathcal{R}_{+}\left(0, \delta_{z}\right)
$$

(see [21]).

Note: if we knew more about energy decay, along the lines of having good estimates for the energy norm

$$
E(t, \lambda)=\|V(t) f\|_{H_{E}(t-\lambda)}=\int_{t-\frac{1}{x}>\lambda}\left(|\nabla V(t) f|^{2}+\left|\frac{\partial V(t) f}{\partial t}\right|^{2}\right) d \operatorname{vol}_{g}
$$

with initial data $f$ compactly supported, we would be able to get better estimates for the decay as $s \rightarrow+\infty$ of $\mathcal{R}_{+}$, and hence drop the mollifier $\phi$ from the statement of this proposition. Friedlander proves in [4] that

$$
\lim _{\lambda \rightarrow \infty} \lim _{t \rightarrow \infty} E(t, \lambda)=0
$$

where $f$ is finite energy initial data; we would need a good deal more however.

\section{Asymptotically hyperbolic Manifolds}

An asymptotically hyperbolic, or conformally compact manifold is a manifold $X$ with boundary equipped with a metric $g$ and defining function $x \geq 0$ such that $x^{2} g=H$ is a smooth metric on $X$, nondegenerate at $\partial X$. We further take $|d x|_{H}=1$ on $\partial X$, which ensures that the sectional curvatures approach -1 at $\partial X$ (see, for instance, [14.)

Notice that $H \uparrow_{\partial X}$ is only defined by $g$ modulo a conformal factor. It is shown in [10, see also [6], that given a conformal representative of $H \uparrow_{\partial X}$ there exists a unique boundary defining function $x$ such that

$$
g=\frac{d x^{2}+h(x, y, d y)}{x^{2}} \text { in } \partial X \times[0, \epsilon) .
$$

From now on fix these coordinates. The definition of the radiation fields will depend on this choice of $x$, or the conformal representative of $H \uparrow_{\partial X}$.

We refer the reader to [10] for a discussion of the Eisenstein function on an asymptotically hyperbolic manifold.

In this setting, we can prove more or less the same results as in the case of scattering manifolds; the analogous results are as follows: 
Theorem 3.1. Let $X$ be a nontrapping asymptotically hyperbolic manifold of dimension $n$.

(1) We have

$$
\mathcal{R}_{+} \in\left(I^{1}\left(\mathbb{R} \times \partial X \times \stackrel{\circ}{X}, \Lambda^{\prime} ; \Omega^{1 / 2}\right), I^{0}\left(\mathbb{R} \times \partial X \times \stackrel{\circ}{X}, \Lambda^{\prime} ; \Omega^{1 / 2}\right)\right),
$$

where $\Lambda_{ \pm}^{\prime}$ is the conic Lagrangian manifold associated to the graph of

$$
\begin{gathered}
\Lambda_{ \pm}=\Lambda_{+} \cup \Lambda_{-} \\
\Lambda_{ \pm}=\left\{z, \zeta, s=\lim _{t \rightarrow \pm \infty} t+\log x\left(\exp _{z}(t \hat{\zeta})\right), \sigma= \pm|\zeta|\right. \\
\left.y=\lim _{t \rightarrow \pm \infty} y\left(\exp _{z}(t \hat{\zeta})\right), \eta_{i}=\lim _{t \rightarrow \pm \infty} \pm x^{-1}|\zeta|\left(h_{0}\right)_{i j}\left(d y^{j} / d x\right)\left(\exp _{z}(t \hat{\zeta})\right)\right\} .
\end{gathered}
$$

(2) Subject to the nondegeneracy assumptions of 2.1 , the symbol of $\mathcal{R}_{+}$is given by (2.7).

(3) Let $E\left(\frac{n}{2}+i \lambda, y, z\right)$ denote the (transpose of the) Eisenstein function. Let $\check{\phi} \in \mathcal{C}_{c}^{\infty}(\mathbb{R})$. Subject to the nondegeneracy assumptions of 2.1 as $|\lambda| \rightarrow \infty$,

$$
\phi(\lambda) * E\left(\frac{n}{2}+i \lambda, y, z\right) \sim \phi(\lambda) * \frac{i}{2 \lambda} \sum_{n=1}^{N} i^{k_{n}} e^{i \lambda S_{n}}\left(\frac{\lambda}{2 \pi i}\right)^{(n-1) / 2}\left|\frac{\partial y^{(n)}}{\partial \hat{\zeta}}\right|^{-1 / 2}
$$

Proof. It is shown in Sá Barreto [20] if $X$ has dimension $n$, and $u=\cos (t \sqrt{\Delta})$ than the rescaled fundamental solution, $v=x^{-(n-1) / 2} u(s-\log x, x, y)$, satisfies

$$
\begin{gathered}
P v \equiv\left(\frac{\partial}{\partial x}\left(2 \frac{\partial}{\partial s}+x \frac{\partial}{\partial x}\right)-x \Delta_{h}+A \frac{\partial}{\partial s}+A x \frac{\partial}{\partial x}+\frac{n-1}{2} A\right) v=0 \\
v \uparrow_{s=\log x}=x^{-(n-1) / 2} \delta(z), \quad \frac{\partial v}{\partial s} \uparrow_{s=\log x}=0, \quad x>0 .
\end{gathered}
$$

The symbol of $P$ is thus $-\left(2 \xi \sigma+x \xi^{2}+x h(x, y, \eta)\right)$, hence the flow is

$$
H_{p}=-2(\sigma+x \xi) \partial_{x}+\left(\xi^{2}+h+x \frac{\partial h}{\partial x}\right) \partial_{\xi}-2 \xi \partial_{s}-x H_{h} .
$$

As in the scattering case the operator $P$ is strictly hyperbolic in $x>0$ and $\{s=\log x\}$ is space-like. Moreover $P$ has an extension $\widetilde{P}$ to a neighborhood of $\{x=0\}$. However, $\widetilde{P}$ is not strictly hyperbolic at $x=0$. So in principle we can only guarantee that $v$ satisfies (2.5), where $\mathcal{L}$ is defined with respect to $P$ in (3.2), when $x>0$.

Since $\widetilde{P}$ can be chosen to be of real principle type, the parametrix construction of the Cauchy problem can be carried though across $\{x=0\}$. This guarantees that in a neighborhood $W$ of $\partial X$ there exists

$$
\widetilde{V_{0}} \in I^{-\frac{1}{4}}(\mathbb{R} \times W \times \stackrel{\circ}{X} ; \mathcal{L})
$$


such that

$$
\begin{gathered}
P \widetilde{V_{0}}=f \in \mathcal{C}^{\infty}(\mathbb{R} \times W) \\
\widetilde{V_{0}} \uparrow_{s=\log x}-\delta(z)=g \in \mathcal{C}_{0}^{\infty}(\stackrel{\circ}{X}), \\
\frac{\partial \widetilde{V_{0}}}{\partial s} \uparrow_{s=\log x}=h \in \mathcal{C}_{0}^{\infty}(\stackrel{\circ}{X}) .
\end{gathered}
$$

Moreover by finite speed of propagation one has that $f$ is supported in $s>s_{0}$, for some $s_{0}$. Again using the hyperbolicity of $P$ in $x>0$, there exists $V \in C^{\infty}(W \cap\{x>0\})$ satisfying

$$
\begin{gathered}
P V=f \quad \text { in } \quad x>0 \\
V \uparrow_{s=\log x}=g, \quad \frac{\partial V}{\partial s} \uparrow_{s=\log x}=h,
\end{gathered}
$$

with $V$ supported in $s>s_{1}$. Since $f$ is supported in $s>s_{0}$ and is smooth up to $\partial X$, and the initial data is compactly supported, the proof of Theorem 2.1 of [20] (in particular, the extension of the energy estimates to the inhomogeneous equation) shows that $V$ has a smooth extension up to $\partial X$.

Therefore the solution to (3.2) satisfies $v \in I^{-\frac{1}{4}}(\mathbb{R} \times X \times \stackrel{\circ}{X} ; \mathcal{L})$ up to $\partial X$.

The transversality of the flow to $\{x=0\}$ and the facts that $\sigma \neq 0$ on the characteristic variety for $x>0$ and $H_{p} \sigma=0$ imply that $v \uparrow_{x=0}$ is a Lagrangian distribution of order 0 on $\mathbb{R} \times \partial X \times \stackrel{\circ}{X}$ with respect to the Lagrangian $\left.\mathcal{L}\right|_{x=0}$, where $\mathcal{L}$ is the flowout of the lift of $N^{*} \Delta \subset T^{*}(\stackrel{\circ}{X} \times \stackrel{\circ}{X})$ to $\Sigma_{p}$. We find in this setting that $\lim _{x \rightarrow 0} x^{-1} d y^{i} / d x=\eta_{j} h_{0}^{i j} / \sigma$, hence $\left.\mathcal{L}\right|_{x=0}$ has the form (3.1).

The remainder of the proof is the same as in the scattering case, using the additional fact from [20] that $E(n / 2+i \lambda, y, z)=-(i / \lambda) \mathcal{F} \mathcal{R}_{+}\left(0, \delta_{z}\right)$.

\section{REFERENCES}

[1] Ivana Alexandrova, Structure of the semi-classical amplitude for general scattering relations, In preparation.

[2] J. J. Duistermaat, Fourier integral operators, Progress in Mathematics, vol. 130, Birkhäuser Boston Inc., Boston, MA, 1996. MR 96m:58245

[3] J.J. Duistermaat and V.W. Guillemin, The spectrum of positive elliptic operators and periodic geodesics, Invent. Math. 29 (1975), 39-79.

[4] F. G. Friedlander, Radiation fields and hyperbolic scattering theory, Math. Proc. Cambridge Philos. Soc. 88 (1980), no. 3, 483-515. MR 82f:35112

[5] Notes on the wave equation on asymptotically Euclidean manifolds, J. Funct. Anal. 184 (2001), no. 1, 1-18. MR 2002k:58059

[6] C. Robin Graham, Volume and area renormalizations for conformally compact Einstein metrics, The Proceedings of the 19th Winter School "Geometry and Physics" (Srní, 1999), no. 63, 2000, pp. 31-42. MR 2002c:53073

[7] V. Guillemin, Sojourn times and asymptotic properties of the scattering matrix, Proceedings of the Oji Seminar on Algebraic Analysis and the RIMS Symposium on Algebraic Analysis (Kyoto Univ., Kyoto, 1976), vol. 12, 1976/77 supplement, pp. 6988. MR 56 \#6759 
[8] Andrew Hassell and Jared Wunsch, The Schrödinger propagator for scattering metrics, Preprint, January 2003.

[9] Mark S. Joshi and Antônio Sá Barreto, Recovering asymptotics of metrics from fixed energy scattering data, Invent. Math. 137 (1999), no. 1, 127-143. MR 2000m:58052

[10] , Inverse scattering on asymptotically hyperbolic manifolds, Acta Math. 184 (2000), no. 1, 41-86. MR 2002g:58052

[11] Jürgen Jost, Riemannian geometry and geometric analysis, third ed., Universitext, Springer-Verlag, Berlin, 2002. MR 2002i:53001

[12] P.D. Lax and R.S. Phillips, Scattering theory, Academic Press, New York, 1967, Revised edition, 1989.

[13] A. Majda, High frequency asymptotics for the scattering matrix and the inverse problem of acoustical scattering, Comm. Pure Appl. Math. 29 (1976), 261-291.

[14] Rafe R. Mazzeo and Richard B. Melrose, Meromorphic extension of the resolvent on complete spaces with asymptotically constant negative curvature, J. Funct. Anal. $\mathbf{7 5}$ (1987), no. 2, 260-310. MR 89c:58133

[15] R.B. Melrose, Spectral and scattering theory for the Laplacian on asymptotically Euclidian spaces, Spectral and scattering theory (Sanda, 1992) (M. Ikawa, ed.), Marcel Dekker, 1994, pp. 85-130.

[16] Geometric scattering theory, Cambridge University Press, 1995.

[17] Richard B. Melrose, Spectral and scattering theory for the Laplacian on asymptotically Euclidian spaces, Spectral and scattering theory (Sanda, 1992), Dekker, New York, 1994, pp. 85-130. MR 95k:58168

[18] V.M. Petkov and L.N. Stoyanov, Sojourn times, singularities of the scattering kernel and inverse problems, MSRI Publications, vol. 47 (2003), Cambridge University Press, to appear.

[19] D. Robert and H. Tamura, Asymptotic behavior of scattering amplitudes in semiclassical and low energy limits, Ann. Inst. Fourier (Grenoble) 39 (1989), no. 1, 155192. MR 91c: 35116

[20] Antônio Sá Barreto, Radiation fields, scattering and inverse scattering on asymptotically hyperbolic manifolds, Preprint.

[21] _ Radiation fields on asymptotically euclidean manifolds, Comm. P.D.E., to appear, Preprint, 2002.

Department of Mathematics, Purdue University, 150 North University Street, WEST LAFAYETTE IN 47907

Department of Mathematics, Northwestern University, 2033 Sheridan Rd., EVANSTON IL 60208 